\title{
Ein militärmedizinisches Examen im Jahre 1794
}

\author{
Von Beat Rüttimann
}

Besonders im klinischen Bereich der Medizin wirkt ein Lehrer am Krankenbett, auf Visiten und bei Fallbesprechungen, in der täglichen Praxis seines Fachgebietes viel nachhaltiger auf Mitarbeiter und Schüler ein als in noch so brillanten Magistralvorlesungen, mit noch so zahlreichen und umfassenden Publikationen, durch noch so auflagenstarke Hand- und Lehrbücher. Veröffentlichungen vermögen seinen Assistenten und Studenten den klinischen Unterricht zu ergänzen; sie vermitteln ein mehr oder weniger systematisches Lehrgerüst und zusätzliche Ausführlichkeit. Vergleiche zwischen unterschiedlichen Lehrmeinungen sind möglich und oftmals ergiebig. Für alle, die einen bestimmten Kliniker nicht selber hören und begleiten konnten, stellt das schriftlich niedergelegte Werk nebst allfälligen Memoiren, weitergereichten Anekdoten und einer lebendigen Tradition den einzigen Zugang zum sachlichen Lehrinhalt und zum ärztlichen Vorbild dar.

In solcher Lage befindet sich der Medizinhistoriker, wenn er sich nicht auf eigene erlebte Vergangenheit berufen kann. Vielleicht ist sogar die Behauptung statthaft, daß im wissenschaftlichen Schrifttum - gerade von besonders anregenden, mitreißenden Lehrern - die Spontaneität des Ausdrucks und die persönliche Eigenart der Formulierung zu «un»gunsten einer geschliffenen und abgewogenen Fassung vermißt wird. Unvermittelte Antworten auf hingeworfene oder in klarer Absicht gestellte Fragen fanden kaum je einen schriftlichen Niederschlag, nicht einmal in Kollegheften, weil die Feder des Hörers den rasch aneinandergefügten Bemerkungen des Dozenten meist nicht zu folgen vermochte. Und vereinzelte Beispiele von besonders schlagfertigen, witzigen oder sarkastischen Reaktionen helfen ebensowenig weiter wie die regelmäßig kolportierten, abgewandelten und ausgeschmückten «Standardantworten» auf notorische Standardfragen.

Können und Wissen eines klinischen Chefs und Lehrers werden im Alltag stets auf die Probe gestellt, stets vor einem kritischen Publikum von Patienten und Personal. Daß aber akademisch ausgewiesene und in leitenden Positionen tätige Ärzte eigentlichen Prüfungen unterworfen würden, ist nicht üblich, auch nicht bei Ärzten der Armee. Allenfalls nimmt eine medizinische Fakultät Bewerber um einen Lehrstuhl in strenge Klausur für die Zeitgenossen unter dem Siegel der Verschwiegenheit und für den 
Historiker unter dem Deckel dürrer, sorgfältig redigierter Sitzungsprotokolle.

\section{Veranlassung}

Anders und als seltene Ausnahme vor bald zweihundert Jahren: «Liberté, Egalité ou la Mort» - die Devise der «einen und unteilbaren», jungen französischen Republik - hatte das Vaterland in Gefahr gebracht. An alle Landesgrenzen wurden eiligst aufgestellte Armeen entsandt, Carnots «levée en masse» der Sansculotten, deren Sanitätsdienst ebenso improvisiert war wie die Ausrüstung, Gliederung und Ausbildung der mit wenigen Veteranen durchsetzten Freiwilligen-Bataillone. Die Zahl der Sanitätsoffiziere - richtiger von Medizinalpersonen, die dem Offiziersstand assimiliert waren mußte im I. republikanischen Jahr verdoppelt und bereits ein Jahr später, 1794, mehr als vervierfacht werden. Eine eigentliche medizinische, zur Hauptsache chirurgische Konskription mit bescheidensten Anforderungen an Wissen und Können! Sofern sie überhaupt bekannt waren, bleiben die Lehrsätze und Denkschriften der vormaligen königlichen Akademie, also gestrige und vorgestrige Meinungen maßgebend. Neuere und aktuelle Erfahrungsberichte ließen noch einige Jahre auf sich warten, erst recht die wichtige und umfangreiche kriegschirurgische Literatur der Kaiserzeit ${ }^{1-4}$.

Eine rigorose Prüfung aller Ärzte der Armee und ihrer Spitäler drängte sich auf. Nicht zuletzt, weil der Bürger-Soldat unter der Trikolore, nach den Losungen der Brüderlichkeit und einer neuen Menschlichkeit, nun für Wunden, Krankheit und Invalidität nicht mehr des Königs Huld, sondern den Staat in Pflicht nehmen konnte. - Von der Beantwortung der «questions épuratoires», welche die Sanitätskommission des Nationalkonvents jedem Militärchirurgen stellte, blieb niemand ausgenommen, nicht einmal der hochangesehene und bestandene Pierre-François Percy (1754-1825) ${ }^{5-10}$.

\section{Der Examinand}

Vornehm, gebildet und belesen, geschichts- und kunstverständig, Doktor der Chirurgie in Besançon mit 21 Jahren, in Paris bevorzugter Schützling des berühmten Louis, schlug Percy eine Laufbahn ein, die seinen Vater bitter enttäuscht und die ihm dieser aufs dringendste abgeraten hatte: Er wurde 


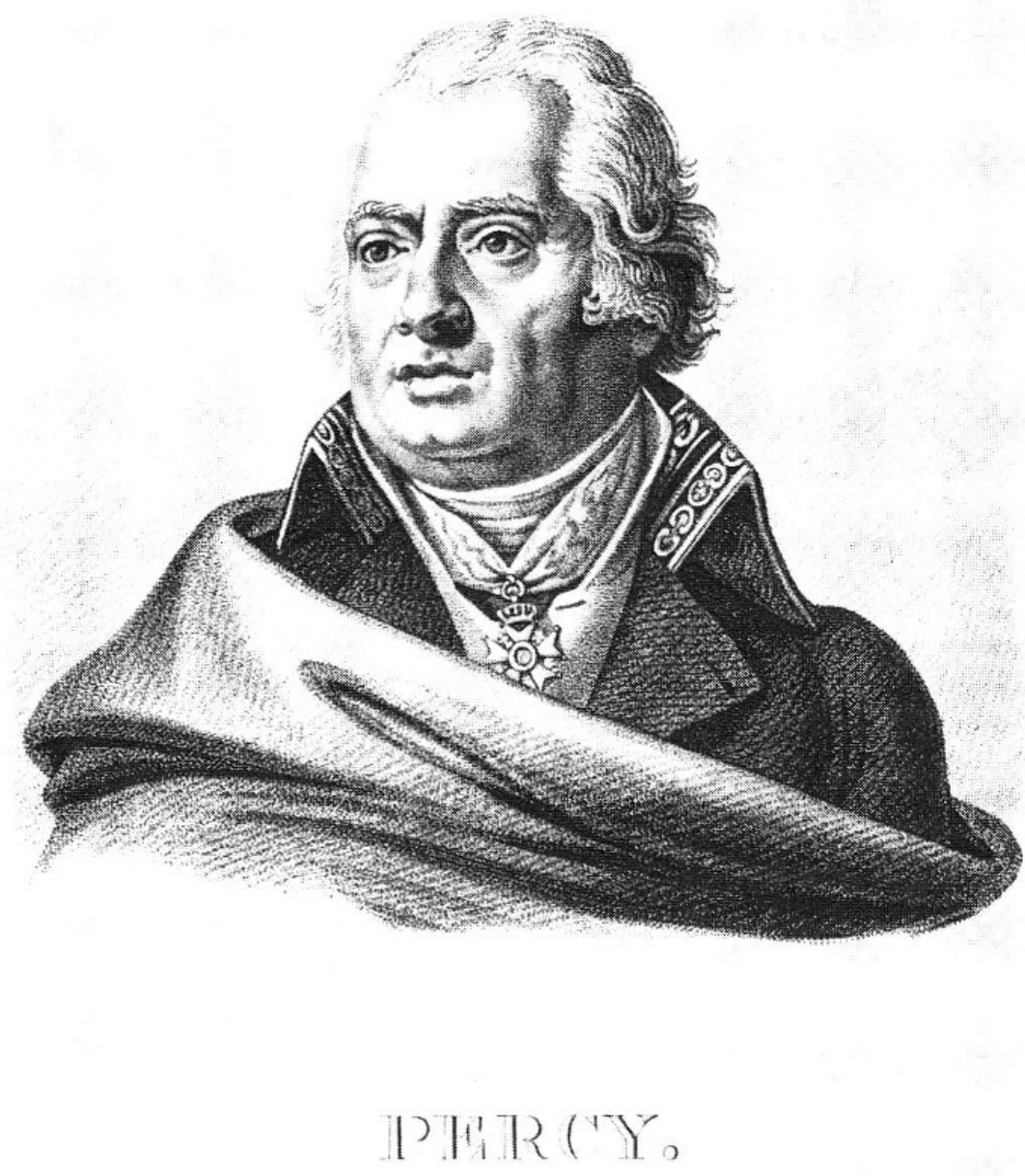

Pierre-François Percy (1754-1825)

Bildersammlung des Medizinhistorischen Instituts der Universität Zürich

Militärarzt, zunächst bei der schottischen Kompanie in Lunéville, dann beim noblen Kavallerieregiment Berry in Béthune. Gleichzeitig setzte er seine Studien fort; viermal in ununterbrochener Folge wurden Percys Arbeiten von der Akademie preisgekrönt, schließlich mit dem Ersuchen, an weiteren Preisausschreiben zur Wahrung der Erfolgsaussichten anderer Kollegen nicht mehr teilzunehmen. Sechzehn wissenschaftliche Auszeich- 
nungen fielen Percy insgesamt zu. 1792, just zu Beginn der Revolutionskriege, veröffentlichte er sein weit verbreitetes Manuel du chirurgien d'armée ${ }^{11}$.

Im Gegensatz zu seinem jüngeren, mindestens ebenso bekannten und tüchtigen Kollegen Dominique Jean Larrey (1766-1842) ${ }^{12}$ war Percy bereits ein reifer Mann, ein geachteter Chirurg und ein erfahrener Militärarzt, als die Französische Revolution ausbrach - mithin keineswegs geprägt oder gar ein Kind dieser umwälzenden Ereignisse. Dennoch wird er im Lauf eines ganzen Vierteljahrhunderts getreulich und selbstlos dienen, trotz lästiger Gebrechen und ernstzunehmender gesundheitlicher Störungen. Trotz seines - in Anbetracht der Strapazen und Entbehrungen - vorgerückten Alters nimmt er an 25 Feldzügen teil, verkraftet vier Verwundungen und vollbringt wahre Heldentaten. Sein hohes Ansehen in der Armee übersteigt sogar die hohe Stellung; beide, Ansehen und Stellung, bewahren ihn aber nicht vor endlosen kleinlichen Querelen und widerlichen Intrigen, gesponnen in der Hauptstadt von der Intendantur und ganz einfach auch von Neidern. Trost und Erholung findet er in der Lektüre der Alten und in der Tiefe seiner starken Persönlichkeit.

\section{Das Examen}

Im September 1794 erwartete Percy, Chefchirurg der Moselarmee, in der Gemeindekanzlei von Bouzonville ein gestempeltes und gesiegeltes Paket aus Paris. Unter Aufsicht der Behörden wurde es geöffnet; es enthielt die Prüfungsfragen, gegliedert in drei Serien. Allein in einem Zimmer, ohne Bücher oder Aufzeichnungen, hatte Percy der Reihe nach alle Fragen zu beantworten. Dieses Werk von 28 Stunden ununterbrochener Arbeit liegt uns glücklicherweise, wenn auch rar, in einem Duodezbändchen von 106 Druckseiten vor ${ }^{13}$, das der nachtragende und nicht besonders umgängliche Autor als Replik auf Unterstellungen und üble Nachrede sofort veröffentlichte. Sein großer Wert liegt im Umstand, daß es in knapper Zeit, in einem Zug und unmittelbar aus dem feldchirurgischen Alltag heraus verfaßt wurde, ohne begründete oder falsche Rücksichten und ohne die zeitübliche Verbrämung - ein eigentliches feldchirurgisches «Extemporale».

Fast scheint es heute, als habe die Sanitätskommission nicht nur examinieren, sondern Auskünfte eines erfahrenen Sanitätsoffiziers einholen wollen. Von Nachforschungen über Bürgertreue keine Spur! Dafür eingehende 
Fragen zu Indikation, Gegenanzeigen und Technik des Débridement, zu dessen extremer Form der primären Amputation, zur Behandlung der Luxationen, zur Gesunderhaltung der Truppe und zur Sanitätstaktik. Auch die Verletzungen, Krankheiten und chirurgischen Eingriffe der Harnwege wurden in diesem Frage-Antwort-Spiel gründlich abgehandelt. Gesamthaft eine verläßliche, berufene Standortbestimmung für die fernab tätige Kommission.

\section{Wundbehandlung und Amputation}

Percys Lehre vom Débridement der Schußwunden entspricht über weite Strecken jetzt noch gültiger Meinung. Erstaunlich sorgfältig geht er auf die Läsionen der einzelnen Gewebe und die Ausdehnung angezeigter Wundrevisionen ein. Für die jahrzehntelange Kontroverse um primäre Amputation und Exartikulation hat Percy - unter Mißachtung königlich-akademischer Empfehlungen und gestützt auf frische kriegschirurgische Erfahrung bereits in groben Zügen eine Lösung gefunden, wie sie sich im Lauf der napoleonischen Kriege und weit darüber hinaus so gut bewähren sollte.

Diese Feststellung dürfen wir uns heute - im Rückblick auf die zweite Hälfte des 18. Jahrhunderts, in Kenntnis der zeitgenössischen Literatur und im Licht von späteren Erfahrungen - bestimmt erlauben ${ }^{14,15}$. Schon Richerand ${ }^{16}$ ist 1825 mehr oder weniger zum selben Schluß gekommen. Doch lagen die Verhältnisse 1794 ganz anders: Aufs heftigste bekämpften sich in Schriften und Pamphleten, in akademischer Rede und sicher auch am Krankenlager die Heißsporne der Amputationschirurgie auf der einen, die allzu konservierenden, die Gliedmaße um jeden Preis erhaltenden Konservativen auf der anderen Seite. Kritik- und maßlos wurden sowohl Indikationen wie Gegenanzeigen zur Amputation gestellt, vor allem auch hinsichtlich des Zeitpunktes für einen (schließlich) als unumgänglich erkannten Eingriff. Im Extremfall langte man wieder bei der Empfehlung von Guy de Chauliac im 14. Jahrhundert an, der geraten hatte, eine brandige Extremität lieber von selber abfallen zu lassen und das Image des Arztes nicht mit derart verstümmelnden Operationen zu belasten ${ }^{17}$.

Es versteht sich wie immer, daß die meisten Chirurgen den wilden Ausschlägen dieses Meinungspendels selten im ganzen Lauf gefolgt sind; standen sie nicht im Zentrum der Diskussionen, nahmen sie oft eine abwartende, nach beiden Seiten kritische Haltung ein und schlossen sich erst 
wohletablierten Lehrsätzen an. Auch das wurde ihnen am Ende des 18. Jahrhunderts nicht einfach gemacht, riet doch beispielsweise der überlegte, in hoher Achtung stehende preußische Generalchirurgus bündnerischer Abstammung Johann Ulrich Bilg(u)er (1720-1796) zum höchst seltenen oder fast gänzlich zu verwerfenden Gebrauch der Gliedmaßenabsetzung ${ }^{18}$. Unter bestimmten, besonders günstigen Umständen mochte sich ein Rettungsversuch lohnen - mit hohem zeitlichem, pflegerischem und ärztlichem Aufwand allerdings, bei immer noch unsicherer Prognose. Diese Voraussetzungen erfüllten sich offenbar für Bilger, denn er konnte über schöne Heilungserfolge berichten. Leitschnur für ärztliches, insbesondere feldchirurgisches Denken hätten sie aber niemals werden dürfen, nicht einmal unter aseptischen Behandlungsbedingungen und in neuester Zeit unter Antibiotika-Schutz. Oft hatte man es besser zu wissen gemeint - oft genug hatte man dafür einen bitteren Preis bezahlt. Am Grundsatz des Débridement hatte auch Bilger nicht gerüttelt, also an Reinigung und Exzision einer komplexen Verletzung, damit sie sich auf einfache Wundverhältnisse reduzierte. Percy faßte in seiner Klausur zusammen: «... en un mot, la plaie rentre dans la classe des plaies simples.»

«Rendre la plaie simple» ist der Grundsatz jeder Art und Ausdehnung von Débridement, bis zur letzten Konsequenz einer vollständigen Abtrennung verletzter Körperpartien, wenn sich anders saubere, sicher verheilende Wundflächen nicht schaffen lassen. Erst an diesem Punkt schreckte Bilger zurück; ihm und seinen Anhängern - die er alle genau kennt und sorgfältig anführt, ebenso sorgfältig wie ihre Gegner im «Amputationslager» - wies Percy aber nach, daß auch sie nicht immer umhin konnten, eine Gliedmaße des Patienten seinem Überleben zu opfern. Unter mißlichen Verhältnissen blieb die primäre Amputation als Débridement unentbehrlich, und je mißlicher die Verhältnisse wurden, desto häufiger war sie angezeigt. Gleichlautende Einsichten steuerten Kriege ${ }^{19}$ und Katastrophen bis auf den heutigen Tag bei. Bilger ist allerdings zugute zu halten, daß er «bloß» die friderizianischen Kabinettskriege in der guten Saison und nicht die soviel unmenschlicher und härter geführten Feldzüge der Revolution, Napoleons und jüngerer Zeiten erlebt hat.

In diesen weittragenden, für die Patienten und ihre Helfer vitalen Auseinandersetzungen besticht das Urteil von Pierre-François Percy durch nüchterne Abgewogenheit und durch den zugrunde gelegten, reichen persönlichen Erfahrungsschatz. Es zeichnet den Weg vor, den bald auch Larrey $^{20-22}$ und nach ihm Generationen von Militärchirurgen beschreiten. 
Sofortige, unmittelbare Amputation oder Exartikulation ist erforderlich bei völliger Zermalmung einer Extremität, bei zirkulärem schwerem Substanzverlust in der Kontinuität (selbst bei intakt gebliebener Blutversorgung), bei Verletzung eines Arterienstammes ohne genügende Umgehungsgefäße, bei offenen Läsionen des Knies und anderer Gelenke und schließlich zur Schaffung günstiger Stumpfverhältnisse, wenn die einwirkende Gewalt die Gliedmaße abgetrennt hat («amputer sur les restes d'un membre»). Unschwer läßt die kurze Aufzählung seitherige Fortschritte der Traumatologie erkennen, indem ganze Kategorien der Indikationsstellung entfallen sind, doch treten sie wieder ins Recht, sobald der zivile Behandlungsstandard nicht mehr gewährleistet ist.

\section{Schultereinrenkung - Badekuren - Hygiene}

Das hippokratische Repositionsmanöver der Schulterluxation lehnte Percy als unnötig qualvoll ab; besonders hart ging er mit Oribasius, Paulus von Aegina, Abulkasim, Chauliac, Scultetus, gar mit Heister und Petit ins Gericht. Von Leitern, Maschinen und anderem Hilfsgerät zur Behandlung der Luxationen wollte er überhaupt nichts wissen. Allenfalls könne man ein gefaltetes Tuch benützen, um geduldig und mit Gefühl eine schonende Einrenkung durch bloße eigene Muskelkraft und die Eigenschwere der Extremität zu bewerkstelligen.

Mit der hippokratischen Einrenkungstechnik der Schulter, aber auch anderer Gelenke ist natürlich die Behandlung der Luxationen auf der eigens dazu konstruierten Bank, dem Scamnum Hippocratis ${ }^{23}$, gemeint. Deshalb sind auch zahlreiche Autoren genannt und mit dem großen, damals noch kaum in Zweifel gezogenen Lehrer ${ }^{24}$ für ein unnötig schmerzhaftes Vorgehen getadelt, weil sie das griechische Therapiegerät ausbauten, weiterentwickelten und der Nachwelt überlieferten. - Tatsächlich hält es schwierig, den großen hippokratischen Aufwand um die Verrenkungen zu verstehen, wenn man sich nicht eine viel weitergefaßte Bedeutung der «Luxation» vor Augen hält. Ganz besonders trifft dies für die Wirbelverrenkung ${ }^{25} \mathrm{zu}$, die aus medizinischer Sicht ja vieles umfassen kann - von kleinsten Verschiebungen, Verkantungen und Wirbelblockierungen der Chiropraktik und der Manualtherapie bis zur schwersten Luxationsfraktur des Achsenorgans. Manche, hauptsächlich gelenknahe Frakturen offenbarten sich früher nur unter dem klinischen Bild einer Verrenkung und erst mit Hilfe der Röntgen- 
strahlen als knöcherner Bruch. Deshalb ist die Auffassung der Griechen und mittelbar ihrer abendländischen Nachfahren leichter und bestimmt auch treffender im Sinne Michlers ${ }^{26}$ zu verstehen: «Für den Hippokratiker war ... jedes gebrochene Glied oder luxierte Gelenk aus seiner Physis, aus seiner natürlichen Lage herausgefallen, und seine Behandlung hatte das Glied oder Gelenk wieder in seine regelrechte Physis, in seine normale Lage zu reponieren.»

Percys Procedere der Schultereinrenkung ist unzweifelhaft sehr schonungsvoll; heutzutage wird es noch unterstützt und erleichtert durch die Anwendung von Muskelrelaxantien und potenten Analgetika. Trotzdem wer wüßte nicht von mühsamster, kaum zu bewerkstelligender Reposition zu berichten, sei es aus technischer oder anatomischer Ursache, so daß schließlich ein ganzes Team zerrte und gegenhielt und der Anästhesist um Maske oder Tubus fürchtete?

Ganz am Schluß der ersten Fragenserie könnte auch ein Kassenvertreter unserer Tage zu Wort gekommen sein: Die Kommission wünschte nämlich Aufschluß über die Zweckmäßigkeit von Badekuren - zweifelsohne mit dem Hintergedanken, einem vermuteten Mißbrauch mit Percys Autorität steuern zu können. In der Tat bestätigte er, während seiner 16jährigen Dienstzeit bei der Berry-Kavallerie noch nie eine Militärperson nach Plombières oder in andere Badeorte geschickt zu haben. Immerhin räumt er auch ein, solche Kuraufenthalte könnten Hydrotherapie und Übungsbehandlung ohne Unterbrüche gewährleisten, daneben Klimawechsel und etwas Zerstreuung - mithin die heutige Argumentation. Doch streng verurteilte er die Willfährigkeit gewisser Sanitätsoffiziere, wirklichen und angeblichen Rheumakranken Badereisen zu verschreiben oder zu gestatten.

Am Beispiel des urologischen Fragenkomplexes zeigt sich besonders deutlich, wie eng zivile und militärische Chirurgie verquickt waren. In der Tat blieben sie seit Ambroise Paré (1510-1590) oftmals schier untrennbar, wie nur wenige leuchtende Namen - Le Dran Vater und Sohn, Petit, Garengeot, Morand, Ravaton und Louis - der führenden französischen Chirurgie bekräftigen mögen. In diese Tradition reiht sich Percy würdig ein.

Fragen der Truppenhygiene und der Krankheitsvorbeugung - etwa Standortwechsel und Wasserversorgung von Feldlagern, Bestattung Gefallener, Absonderung venerisch Erkrankter und sonst ansteckender Krankheitsfälle - wurden mit Medizinern und Apothekern abgesprochen. Auch unter ihnen fanden sich, trotz der weit geringeren Zahl, zu aller Zeit bewährte Männer. 


\section{Sanitätstaktik}

Einzig die sanitätsdienstliche Taktik hielt nicht ganz Schritt; noch immer fußte sie auf Reglementen des «Guten Königs» Henri IV und seines Ministers Sully. Den Kriegszügen der Kabinette und der stehenden Heere war sie noch einigermaßen gewachsen, aber keinesfalls den neuen Volkskriegen und dem wuchtigen Einsatz des Feuers. Nach zweijähriger Dienstzeit im Feld schlug Percy ein Näherrücken der Ambulanz an das Kampfgeschehen vor. Zuhanden der Kommission umschrieb er sehr genau die Aufgaben und Pflichten des Sanitätspersonals; er nutzte die günstige Gelegenheit, Aufgeschlossenheit und Bürgertugend unter Beweis zu stellen.

Später bildete er mobile Patrouillen, aufgesessen auf länglichen Munitionskarren der Artillerie, «Wurst» genannt und reichlich ausgestattet mit Instrumenten und Verbandmaterial. Krankenträger oder «despotats» ${ }^{27}$ wurden mit einer halben Bahre (in Einzelteilen: Schaft der Lanze, auf den Tornister geschnalltes Querstück und eingerolltes Tuch) ausgerüstet und in Kompanien gegliedert. Doch als beweglicher und zweckmäßiger erwiesen sich die «Fliegenden Ambulanzen» Dominique Jean Larreys, die nach und nach Percys Organisation verdrängten. Der junge Larrey hatte noch mehr Schwung und revolutionären Elan! In der Kaiserzeit werden sie dann beide an zahlreichen Feldzügen in alle Winkel Europas teilnehmen, sich gelegentlich etwas gram sein und sich schneiden, doch rasch wieder versöhnt die Auszeichnung Napoleons verdienen, der sie «la Providence du soldat» nannte.

\section{Nur eine müßige Fragerei?}

Im zweiten Drittel der Prüfung war Percy auch aufgetragen, für Chirurgen erster und dritter Klasse einen ähnlichen Fragenkatalog auszuarbeiten. Er konnte sich nicht verkneifen, etwas giftig - hier mit den Worten von Beppina Freifrau von Weinbach ${ }^{28}$, der Übersetzerin seines Feldzugs-Journals einzuleiten: «Die Kommission weiß sehr wohl, wie wenig Geist dazu nötig ist, Fragen zu stellen, und machte mich zum Examinator, ohne Zweifel deshalb, um mich etwas zu Atem kommen zu lassen.»

Allzu ungeschickt hat sich die Behörde ihrer Aufgabe und ihres Anliegens nicht entledigt, wenngleich Percy die vorgelegten Fragen - und damit wohl die ganze Prüfung - großenteils spitzfindig und unnütz fand. Heute sind wir 
der Kommission verpflichtet. Auch uns hat sie ein Gespräch mit der unbestrittenen Autorität jener Zeit vermittelt und trefflich die Themen ausgewählt, die uns am meisten ansprechen - und stets noch beschäftigen.

\section{Quellennachweis}

${ }^{1}$ Bégin, L.-J.: Etudes sur le Service de Santé militaire en France, son passé, son présent, son avenir. Chapitre II: de 1792 à 1834. J.-B. Baillière, Paris 1849

${ }^{2}$ Brice et Bottet: Le Corps de Santé militaire en France. Son évolution - ses campagnes 1708-1882. Berger-Levrault, Paris et Nancy 1907

${ }^{3}$ Huard, P.: Sciences - Médecine - Pharmacie de la Révolution à l'Empire (1789-1815). Roger Dacosta, Paris 1970

${ }^{4}$ Chevalier, A.G.: Probleme des Sanitätswesens in den Napoleonischen Heeren. Ciba Zeitschrift 7 (1940) 2582

${ }_{5}^{5}$ Busquet, P. et Gilbert, A./Genty, M.: Le baron Percy (Pierre-François), 28 Octobre 1754-18 Février 1825. Inspecteur Général du Service de Santé militaire, Professeur à la Faculté de Médecine de Paris, Membre de l'Académie Royale de Médecine, Membre de l'Institut. Les Biographies Médicales, Tome II. J.-B. Baillière et fils, Paris o. J.

${ }^{6}$ Dictionnaire historique de la Médecine ancienne et moderne (par Dezeimeris). Percy (Pierre François). Tome III. $1^{\text {re }}$ partie. Béchet jeune, Paris 1836

7 Dictionnaire encyclopédique des Sciences médicales (Directeur: Amédée Dechambre). Percy (Pierre-François). Tome vingt-deuxième. P. Asselin et G. Masson, Paris 1886

${ }^{8}$ Pariset, E.: Histoire des membres de l'Académie Royale de Médecine ou recueil des éloges lus dans les séances publiques de l'Académie Royale de Médecine. Eloge de M. le Baron Percy, lu dans la séance publique du 28 février 1828. Tome premier. J.-B. Baillière, Paris 1845

${ }^{9}$ Rochard, J.: Histoire de la Chirurgie française au XIX ${ }^{\mathrm{e}}$ siècle. Etude historique et critique sur les progrès faits en chirurgie et dans les sciences qui s'y rapportent depuis la suppression de l'Académie Royale de Chirurgie jusqu'à l'époque actuelle. J.-B.Baillière et fils, Paris 1875

${ }^{10}$ Tournier-Lasserve, Ch.: Les chirurgiens militaires et l'Académie de Médecine. Bull. Acad. Nat. Méd. 166 (1982) 601

11 Percy, P.-F.: Manual du Chirurgien-d'Armée, ou Instruction de Chirurgie-militaire. Méquignon l'aîné, Paris 1792

12 Triaire, P.: Dominique Larrey et les Campagnes de la Révolution et de l'Empire. Alfred Mame et fils, Tours 1902. - Das Standardwerk über D. J. Larrey. Siehe auch ${ }^{15}$ mit weiteren Angaben und Quellennachweisen.

${ }_{13}$ Percy, P.-F.: Réponses du citoyen Percy, Chirurgien en chef de l'armée de la Moselle, aux questions épuratoires qui lui ont été proposées par la Commission de Santé séante à Paris. Collignon, Metz $\mathrm{III}^{\mathrm{e}}$ année républicaine

14 Ackerknecht, E. H.: Pariser Chirurgie von 1794 bis 1850. Gesnerus 17 (1960) 137

15 Rüttimann, B.: Larreys Amputationstechnik. Gesnerus 36 (1979) 140

${ }^{16}$ Richerand, A.-B.: Histoire des Progrès récens (sic) de la Chirurgie. Béchet jeune, Paris 1825 
17 Nicaise, E.: La Grande Chirurgie de Guy de Chauliac... composée en l'an 1363. Félix Alcan, Paris 1890

${ }^{18}$ Bilguer, J. U.: Abhandlung von dem sehr seltenen Gebrauch, oder, der beynahe gänzlichen Vermeidung des Ablösens der menschlichen Glieder, aus dem Lateinischen übersetzt und mit noch einigen Wahrnehmungen vermehrt. Arnold Weber, Berlin 1761

${ }^{19}$ Dubs, J.: Die Feldchirurgie im schweizerischen Gefechts-Sanitätsdienst. I. Einführung in die Geschichte der Kriegschirurgie und des Heeres-Sanitätswesens S.1-85. Morgartenverlag, Zürich und Leipzig 1939

${ }^{20}$ Larrey, D. J.: Relation historique et chirurgicale de l'expédition de l'Armée d'Orient, en Egypte et en Syrie. Demonville et sœurs, Paris 1803

${ }^{21}$ Larrey, D. J.: Mémoires de Chirurgie militaire et Campagnes. Tome I-IV. J. Smith (et F. Buisson), Paris 1812/1817

${ }^{22}$ Larrey, D. J.: Relation médicale de campagnes et voyages, de 1815 à 1840. J.-B. Baillière, Paris 1841

${ }^{23}$ Lewek, W. R.: Die Bank des Hippokrates. Ein Beitrag zur Geschichte der Behandlung von Frakturen und Luxationen. Janus 4 (1936) 1-28, 65-84

${ }^{24}$ Ackerknecht, E. H.: Histoire de la médecine, pourquoi? Médecine et Hygiène 18 (1960) 580

${ }^{25}$ Rüttimann, B.: Hippokratische «Wirbelverrenkung». Gesnerus 40 (1983) 159

${ }^{26}$ Michler, M.: Kniegelenk und Wirbelsäule in den beiden hippokratischen Schriften «Über die Knochenbrüche» und «Über die Gelenke». Orthop. Praxis 5 (1969) 209

${ }^{27}$ Percy, P.-F.: Despotats. Dictionaire (sic) des sciences médicales. Band 8, S.569-579 und 4 Tafeln. C. L.F. Panckoucke, Paris 1814

${ }^{28}$ Longin, E. (übersetzt von Beppina Freifrau von Weinbach): Feldzugs-Journal des Baron Percy, Chefchirurg der großen Armee (1754-1825). Heinrich Schmidt \& Carl Günther, Leipzig 1906

\section{Summary}

A curious examination of all military surgeons took in 1794 place in Revolutionary France. Even the famous Pierre-François Percy had to undergo it. His answers are-because of their spontaneity - of the highest interest for the history of military medecine as well as for problems of our days.

PD Dr. med. Beat Rüttimann

Orthopädische Universitätsklinik Balgrist

Forchstraße 340

CH-8008 Zürich

Medizinhistorisches Institut der Universität Zürich

Rämistraße 71

CH-8006 Zürich 